\title{
Prefacio Geología del Valle de México
}

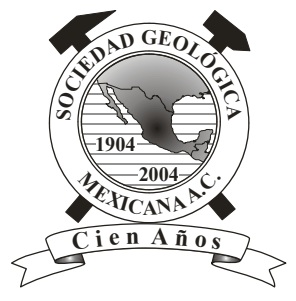

La Zona Metropolitana de la Cuenca de México alberga casi el $20 \%$ de la población del país, con una densidad de más de 2500 habitantes por kilómetro cuadrado y concentra la mayor parte de la actividad económica y política del país. Durante los años 70's a 90's experimentó un acelerado aumento de la densidad de población y de expansión urbana, lo que ha ocasionado un incremento desproporcionado en la demanda de servicios urbanos como agua, transporte, energía, saneamiento, etc. Este proceso de urbanización acelerada en forma gradual y continua ha sido más rápido que el aumento en la capacidad para cubrir la demanda de servicios urbanos y de infraestructura urbana, lo que ha generado enormes problemas para la disposición de basura y agua residuales. Ello genera daños económicos y ambientales que ya se muestran dramáticos en términos de inundaciones, tránsito vehicular, uso del suelo, clima local, y contaminación del agua, suelo y aire, además de los riesgos asociados a la geología de la cuenca como sismicidad, vulcanismo, deslizamiento de laderas, etc.

La atención en muchos de estos problemas está relacionada con el conocimiento sobre la dinámica de los procesos geológicos, la disponibilidad y agotamiento de los recursos naturales, el grado de deterioro de las fuentes, el desequilibrio generado en los sistemas naturales. Por esta razón, en el presente volumen especial del Boletín de la Sociedad Geológica Mexicana denominado "Geología del Valle de México "se presenta una integración de los trabajos científicos más recientes sobre la Cuenca de México con la finalidad de que estos conocimientos estén disponibles para todo público, en especial de los responsables de la toma de decisiones, analizando el pasado, el presente y el futuro de la zona metropolitana dentro de la Cuenca de México.

Este número especial incluye la reconstrucción de la historia de los cambios ambientales y climáticos registrados en las secuencias sedimentarias lacustres en la zona del Lago de Chalco, para analizar la recurrencia de la actividad volcánica de la región (Ortega-Guerrero et $a l$.) y con la caracterización del paleoambiente del Eoceno tardío, para documentar la paleoflora y su composición, lo que permitirá establecer la presencia de cuerpos de agua a través de palinomorfos indicadores de ambientes lacustres (Lozano-García y Sosa Nájera). Con respecto a las condiciones ambientales recientes, la simulación computacional del tiempo meteorológico en la zona metropolitana en los últimos 500 años permite estimar el impacto de la urbanización y la desaparición del sistema lacustre en el régimen térmico de la Cuenca de México (Ruiz-Angulo y López-Espinoza). La reconstrucción de la distribución temporal y espacial de la cubierta de suelos del pasado permite entender su relación con la estructura actual de suelos y sedimentos en un sector semi-árido de la cuenca de México (Solleiro-Rebolledo et al.), mientras que las condiciones hidrológicas son evaluadas mediante la determinación de los factores que inciden en la vulnerabilidad de las fuentes de abastecimiento de agua potable a la zona metropolitana (Escolero et al.). Asimismo se presenta un trabajo sobre sitios de interés geológico y geomorfológico del Patrimonio de la Reserva Ecológica del Pedregal de San Angel y áreas cercanas, ubicados al sur de la Cuenca de México, con el fin de conservar un espacio de gran valor biológico y cultural (Palacio-Prieto y Guibalud). En otro grupo de trabajos se caracteriza el medio físico en que se ubica el área metropolitana mediante el estudio de procesos erosivos fluviales en la zona del Popocatépetl y su relación con su intensa actividad volcánica (Castillo et al.), las características de la estructura cortical somera por debajo de la Sierra Chichinautzin y su relación con la sismicidad en la cuenca (Campos-Enriquez et al.), y un análisis de la posible correlación de las unidades del subsuelo profundo de la Cuenca de México con el registro estratigráfico del Cenozoico anterior a la Faja Volcánica Trans-Mexicana en la parte norte de la Sierra Madre del Sur (González-Torres et al.). Algunos aspectos hidrogeológicos son analizados mediante el estudio de la relación de subsidencia del terreno y el abatimiento del nivel estático en pozos debido a la extracción de agua subterránea en la zona metropolitana (Solano-Rojas et al.), y el estudio de los factores participantes en la composición hidrogeoquímica del agua de abastecimiento a pobladores de la Delegación Iztapalapa (Domínguez-Mariani et al.). En un grupo adicional de trabajos se exploran otras posibles fuentes de abastecimiento de agua para la zona metropolitana, con el estudio de la geología y estratigrafía del pozo profundo San Lorenzo Tezonco y del sur de la Cuenca de México (Arce et al.) y del estudio de la evolución de las pérdidas hidráulicas de pozo durante el desarrollo y aforo del pozo San Lorenzo Tezonco (Morales-Casique et al.). Con vista al futuro, se proponen opciones para el manejo del exlago de Texcoco en favor del desarrollo económico y ambiental de la zona metropolitana a largo plazo (Jazcilevich-Diamant et al.).

Indudablemente, con estos trabajos no se cubren todos los tópicos relacionados con las Ciencias de la Tierra para la Cuenca de México, ya que existen otro temas interesantes como inundaciones, actividad volcánica reciente, sismos frecuentes, manejo de aguas residuales, etc. Sin embargo, 
este conjunto de trabajos representa un primer paso para la integración del conocimiento disponible sobre Ciencias de la Tierra en la Cuenca de México y esperamos que dé lugar a otros trabajos que aporten otros conocimientos a las problemáticas de la zona metropolitana.

Óscar A. Escolero Eric Morales-Casique José L. Arce 